\title{
Updated global analysis of Higgs couplings
}

\author{
John Ellis ${ }^{a, b}$ and Tevong You ${ }^{a}$ \\ ${ }^{a}$ Theoretical Particle Physics and Cosmology Group, Physics Department, \\ King's College London, London WC2R 2LS, U.K. \\ ${ }^{b}$ TH Division, Physics Department, CERN, \\ CH-1211 Geneva 23, Switzerland \\ E-mail: john.ellis@cern.ch, tevong.you@kcl.ac.uk
}

ABSTRACT: There are many indirect and direct experimental indications that the new particle $H$ discovered by the ATLAS and CMS Collaborations has spin zero and (mostly) positive parity, and that its couplings to other particles are correlated with their masses. To a high degree of confidence, it is a Higgs boson, and here we examine the extent to which its couplings resemble those of the single Higgs boson of the Standard Model. Our global analysis of its couplings to fermions and massive bosons determines that they have the same relative sign as in the Standard Model. We also show directly that these couplings are highly consistent with a dependence on particle masses that is linear to within a few \%, and scaled by the conventional electroweak symmetry-breaking scale to within $10 \%$. We also give constraints on loop-induced couplings, on the total Higgs decay width, and on possible invisible decays of the Higgs boson under various assumptions.

Keywords: Higgs Physics, Technicolor and Composite Models, Standard Model

ARXIV EPRINT: 1303.3879 


\section{Contents}

1 Introduction and summary 1

2 Summary of the data $\quad 2$

3 Higgs couplings to bosons and fermions 5

4 Probing the mass dependence of Higgs couplings $\quad 9$

5 The total Higgs decay rate 11

6 Conclusions 13

\section{Introduction and summary}

It has now been established with a high degree of confidence that the new particle $H$ with mass $\sim 126 \mathrm{GeV}$ discovered by the ATLAS [1, 2] and CMS [3, 4] has spin zero and (mainly) positive-parity couplings, as expected for a Higgs boson [5, 6]. Minimal spintwo alternatives with graviton-like couplings have been disfavoured by measurements of the $H$ couplings to vector bosons [7], and quite strongly excluded by constraints on the energy dependence of $H$ production [8]. The graviton-like spin-two hypothesis has also been disfavoured strongly by analyses of $H$ decays into $\gamma \gamma[9], Z Z^{*}$ and $W W^{*}$ final states [10, 11], and the positive-parity assignment is favoured by decays into $Z Z^{*}$ [12], in particular. ${ }^{1}$ To a high degree of confidence, the $H$ particle is a Higgs boson.

In this paper we make updated global fits to the $H$ couplings to other particles with the aim of characterizing the extent to which they resemble those of the Higgs boson of the Standard Model. There has been considerable progress since our previous analysis of $H$ couplings [59], including updates at the Hadron Collider Physics conference in November 2012 [17], the CERN Council in December 2013 [18, 19], the Moriond Electroweak Conference [20] and the Aspen 'Higgs Quo Vadis' Meeting in March 2013 [21], and most recently an update of the CMS $H \rightarrow \gamma \gamma$ data at the Moriond QCD session [22].

There have been many analyses of the $H$ couplings [23-59], some also including the Moriond 2013 data $[60,61] .{ }^{2}$ Many of these analyses, including those made by the different experimental Collaborations, assume simple parameterizations in which the couplings of the Standard Model Higgs boson to bosons and fermions are rescaled by factors $a_{V}$ and $c_{f}$, respectively (or equivalently by factors $\kappa_{V, f}$ ) [64]. Fits with non-minimal couplings to

\footnotetext{
${ }^{1}$ It is also impressive that the mass of the $H$ particle coincides with the best fit for the mass of the Higgs boson found in a global fit to precision electroweak data taking account of pre-LHC searches at LEP and the TeVatron [13], and is also highly consistent with low-energy supersymmetry [14-16].

${ }^{2}$ After this work was completed ATLAS and CMS have made public their couplings analyses [62, 63].
} 
massive vector bosons have also been considered, as have fits in which the loop-induced couplings to gluons and photons deviate by factors $c_{g, \gamma}$ from the values predicted in the Standard Model. The latter have been of interest in view of the possible excess of $H \rightarrow \gamma \gamma$ decays relative to the Standard Model prediction, particularly as reported by the ATLAS Collaboration [9]. Since the $H \gamma \gamma$ coupling could in principle receive contributions from new massive charged particles, and the $\mathrm{Hgg}$ coupling from new massive coloured particles, these are particularly sensitive to new physics beyond the Standard Model. In this paper we make updated global fits to the $H$ couplings within such common phenomenological frameworks.

We also revisit parameterizations of the $H$ couplings to fermions and bosons that were first considered in [59], which are designed specifically to probe the dependence of the $H$ couplings on particle masses. Namely, we consider parameterizations of the $H$ couplings to fermions $\lambda_{f}$ and massive bosons $g_{V}$ of the form

$$
\lambda_{f}=\sqrt{2}\left(\frac{m_{f}}{M}\right)^{1+\epsilon}, g_{V}=2\left(\frac{m_{V}^{2(1+\epsilon)}}{M^{1+2 \epsilon}}\right),
$$

which reduce to the couplings of the Standard Model Higgs boson in the double limit $\epsilon \rightarrow$ $0, M \rightarrow v=246 \mathrm{GeV}$. This parameterization addresses explicitly the question the extent to which the $H$ particle resembles a quantum excitation $[5,6]$ of the Englert-Brout-Higgs field that is thought to give masses to the particles of the Standard Model [5, 6, 65-67].

We find that, in the absence of contributions from any particles beyond the Standard Model, a combination of the Higgs signal strengths measured in different channels is now very close to the Standard Model value, within $13 \%$ at the $68 \% \mathrm{CL}$. We also find, for the first time, a strong preference for the couplings to bosons and fermions to have the same sign, also as expected in the Standard Model, driven largely by the new CMS result on $H \rightarrow \gamma \gamma$ decay. This also means that there is no significant evidence of additional loop contributions to the $H \gamma \gamma$ beyond those due to the top quark and the $W$ boson. Using the parameterization (1.1), we find that the dependence of the Higgs couplings to different particle species is within a few $\%$ of a linear dependence of their masses. Within the parameterization (1.1), or marginalizing over the $H$ couplings to Standard Model bosons and fermions, we find that the total Higgs decay rate lies within $20 \%$ of the Standard Model value at the $68 \%$ CL. If the couplings of the Higgs Boson to Standard Model particles have their Standard Model values and there are no non-standard contributions to the $H g g$ and $H \gamma \gamma$ amplitudes, the upper limit on invisible Higgs decays is $10 \%$ of the total Higgs decay rate.

\section{Summary of the data}

The analysis of this paper is based mainly on the material presented by the LHC and Tevatron experimental Collaborations at the March 2013 Moriond Conferences in La Thuile [20, 22]. The following are some of the main features of interest among the new results:

- The $H \rightarrow \bar{b} b$ signal strength reported by the TeVatron experiments has reduced from $2.0 \pm 0.7$ to $1.6 \pm 0.75$ times the Standard Model value. 
- A new $H \rightarrow \tau^{+} \tau^{-}$result of $1.1 \pm 0.4$ has been reported by CMS, improving on the previous value of $0.7 \pm 0.5$.

- The $H \rightarrow \gamma \gamma$ signal strength reported by ATLAS has reduced somewhat from $1.80_{-0.36}^{+0.4}$ to $1.65_{-0.30}^{+0.34}$ times the Standard Model value. Most importantly, CMS has reported a new result of $0.78_{-0.26}^{+0.28}$ for the signal strength using an MVA approach.

- The $H \rightarrow W W^{*}$ signal strength reported by ATLAS has reduced from $1.5 \pm 0.6$ to $1.01 \pm 0.31$ times the Standard Model value.

All the latest available results from ATLAS, CMS and TeVatron are incorporated into our global fit. The experimental data are used to reconstruct the likelihood in a combination of three possible ways according to the available information: 1) using the official best-fit central value of $\mu$ with its $1-\sigma$ error bars, 2) using the given number of signal, background and observed events with their respective errors, or 3) reconstructing the central value of $\mu$ from the $95 \%$ CL expected and observed $\mu$. Specifically, the data inputs are as follows:

- The TeVatron $H \rightarrow \bar{b} b, \tau^{+} \tau^{-}, W W^{*}, \gamma \gamma$ combined best-fit $\mu$ and $1-\sigma$ error bars from [68].

- The likelihood for the CMS $8 \mathrm{TeV} W W^{*} 0,1$-jet analysis is reconstructed from the numbers of events given in table 4 of [69]. The $W W^{*} 2$-jet event numbers are instead taken from table 3 of [70]. In addition, we use the fit values from [71] for the $7-\mathrm{TeV}$ CMS $W W^{*}$ data. The ATLAS Collaboration provides 0,1 -jet and 2-jet $\mu$ central values and $1-\sigma$ ranges for a combination of 7 - and $8-\mathrm{TeV}$, which we treat effectively as $8 \mathrm{TeV}$. The percentages of the vector-boson fusion (VBF) production mode contributions to the signals in the 0,1 and 2 -jet channels are taken to be $2 \%, 12 \%$ and $81 \%$, respectively [72].

- For $H \rightarrow b \bar{b}$ in CMS we used the 7- and $8-\mathrm{TeV}$ best-fit values from [71] and [73], while for ATLAS the likelihood was reconstructed from the 95\% CL expected and observed values of $\mu$ at 7 and $8 \mathrm{TeV}$ given in [74].

- The CMS $H \rightarrow \tau^{+} \tau^{-}$and $Z Z^{*}$ and $Z Z^{*}$ dijet rates were taken from the central values given in [10]. Since no separate 7 - and $8-\mathrm{TeV}$ numbers are given for these, we treat them effectively as $8 \mathrm{TeV}$. Numbers of events for the ATLAS $H \rightarrow Z Z^{*} 7$ and $8-\mathrm{TeV}$ analyses are provided separately in [10], while the ATLAS $H \rightarrow \tau^{+} \tau^{-}$ likelihood is reconstructed using the $95 \%$ expected and observed values of $\mu$ given in [75]. The $\operatorname{VBF} \tau^{+} \tau^{-}$efficiencies are taken from [76].

- The CMS $\gamma \gamma$ central values are given for six (five) different subchannels at 8 (7) TeV in [10], along with the percentage contributions from all production mechanisms in table 2 in [77]. The same information can be found for ATLAS at $7 \mathrm{TeV}$ in $[1,2]$ and at $8 \mathrm{TeV}$ in [10], broken down into eleven subchannels including two VBF-dominated ones. The CMS update is reported for a cut-based and MVA analysis; we use the MVA result, which has the greater sensitivity. 
The likelihood is assumed to have a Gaussian distribution, which is in practice a good approximation for a substantial number of events $\gtrsim 10$. In cases where asymmetric errors are reported, the larger of the two is conservatively taken to be the symmetric $1 \sigma$ error. Due to the limited experimental information available, we ignore correlation effects and any marginalization over nuisance parameters, which is not expected to affect our results outside the $\sim 10 \%$ current level of accuracy. For each individual experiment we have checked that our combinations of the likelihoods for the various subchannels agree with official combinations with only slight exceptions, for example the CMS $7-\mathrm{TeV} \gamma \gamma$ analysis $(\mu=$ $1.58_{-0.61}^{+0.60}$ instead of the official value of $1.69_{-0.59}^{+0.65}$ ). When combined with the CMS 8-TeV data (for which we reproduce the official central value) we calculate for the combined CMS $\gamma \gamma$ data a value of $\mu=0.72_{-0.26}^{+0.24}$ (to be compared with the official value of $0.78_{-0.26}^{+0.28}$ ). This difference of a fraction of the quoted error does not impact significantly our overall results.

As a preliminary to our analysis, we compile in figure 1 the overall signal strengths in the principal channels, as calculated by combining the data from the different experiments. Thus, for example, in the first line we report the $V+(H \rightarrow \bar{b} b)$ signal strength found by combining the data on associated $V+H$ production from the TeVatron and LHC. As can be seen in the second line, so far there is no significant indication of associated $\bar{t} t+H$ production. The third line in figure 1 combines the experimental information on the $H \rightarrow \bar{b} b$ signal strengths in these two channels. Signals for $H \rightarrow \tau^{+} \tau^{-}$decay have now been reported in various production channels, as reported in the next three lines of figure 1 , and the combined signal strength is given in the following line. As we have discussed, data are available on $H \rightarrow \gamma \gamma$ final states following production in gluon-gluon collisions and via vector-boson fusion. The central values of the corresponding signal strengths are now only slightly larger than the Standard Model predictions, and we return later to a discussion of the significance of these measurements. The signal strengths in the $H \rightarrow W W^{*}$ and $Z Z^{*}$ final states are very much in line with the predictions of the Standard Model. These dominate the determination of the combined signal strength reported in the last line of figure 1, together with the $\gamma \gamma$ final state. It is striking that the available data already constrain the combined Higgs signal strength to be very close to the Standard Model value:

$$
\mu=1.02_{-0.12}^{+0.11}
$$

We present separately the combined signal strength in the VBF and VH channels without the loop-induced $\gamma \gamma$ final state, which lies slightly (but not significantly) above the Standard Model value. To the extent that a signal with direct Higgs couplings in both the initial and final state is established, this combination disfavours models that predict a universal suppression of the Higgs couplings. ${ }^{3}$

\footnotetext{
${ }^{3}$ We address later in a full fit of the effective couplings of the Higgs to photons and gluons the question whether an enhancement of the loop-induced gluon fusion production could compemsate for this by contaminating the VBF cut selection.
} 


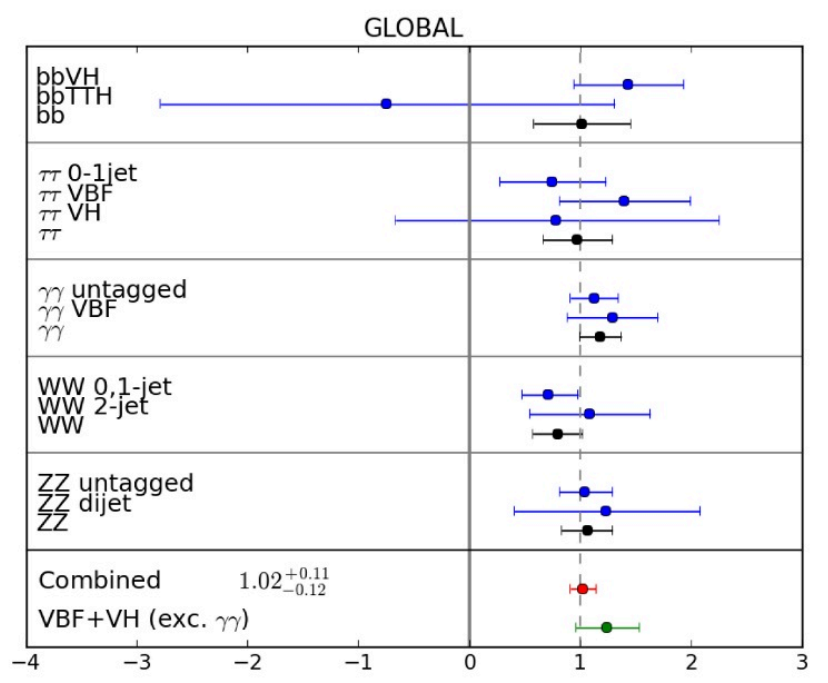

Figure 1. A compilation of the Higgs signal strengths measured by the ATLAS, CDF, D0 and CMS Collaborations in the $\bar{b} b, \tau^{+} \tau^{-}, \gamma \gamma, W W^{*}$ and $Z Z^{*}$ final states. We display the combinations of the different channels for each final state, and also the combination of all these measurements, with the result for the VBF and VH channels (excluding the $\gamma \gamma$ final state) shown separately in the bottom line.

\section{Higgs couplings to bosons and fermions}

Our first step in analyzing the implications of these data uses the following effective lowenergy nonlinear Lagrangian for the electroweak symmetry-breaking sector [78-81]:

$$
\begin{aligned}
\mathcal{L}_{\mathrm{eff}}= & \frac{v^{2}}{4} \operatorname{Tr}\left(D_{\mu} U D^{\mu} U^{\dagger}\right) \times\left[1+2 a \frac{H}{v}+\ldots\right] \\
& -\frac{v}{\sqrt{2}} \Sigma_{f} \bar{f}_{L} \lambda_{f} f_{R}\left[1+c_{f} \frac{H}{v}+\ldots\right]+\text { h.c. }
\end{aligned}
$$

where $U$ is a unitary $2 \times 2$ matrix parametrizing the three Nambu-Goldstone fields that give masses to the $W^{ \pm}$and $Z^{0}$ bosons, $H$ is the physical Higgs boson field and $v \sim 246 \mathrm{GeV}$ is the conventional electroweak symmetry-breaking scale. The coefficients $\lambda_{f}$ are the Standard Model Yukawa couplings of the fermion flavours $f$, and the factors $a$ and $c_{f}$ characterize the deviations from the Standard Model Higgs boson couplings of the $H$ couplings to massive vector bosons and the fermions $f$, respectively. The couplings of the Higgs boson to massless boson pairs $g g$ and $\gamma \gamma$ are described by the following dimension-5 loop-induced couplings:

$$
\mathcal{L}_{\Delta}=-\left[\frac{\alpha_{s}}{8 \pi} c_{g} b_{g} G_{a \mu \nu} G_{a}^{\mu \nu}+\frac{\alpha_{e m}}{8 \pi} c_{\gamma} b_{\gamma} F_{\mu \nu} F^{\mu \nu}\right]\left(\frac{H}{V}\right),
$$

where the coefficients $b_{g, \gamma}$ are those found in the Standard Model, and the factors $c_{g, \gamma}$ characterize the deviations from the Standard Model predictions for the $H$ couplings to massless vector bosons. 
One specific model for a common rescaling factor of all fermion and vector boson Higgs couplings is a minimal composite Higgs scenario [78-81], the MCHM4, in which the compositeness scale $f$ is related to $(a, c)$ by

$$
a=c=\sqrt{1-\left(\frac{v}{f}\right)^{2}} .
$$

A similar universal suppression is found in pseudo-dilaton models. A variant of this minimal model with a different embedding of the Standard Model fermions in $\mathrm{SO}(5)$ representations of the new strong sector, the MCHM5, has separate vector and fermion rescalings:

$$
a=\sqrt{1-\left(\frac{v}{f}\right)^{2}} \quad, \quad c=\frac{1-2\left(\frac{v}{f}\right)^{2}}{\sqrt{1-\left(\frac{v}{f}\right)^{2}}} .
$$

In the following we confront the data with these specific models, as well as an 'anti-dilaton' scenario in which $c=-a$.

Figure 2 compiles the constraints imposed by the data summarized in figure 1 on the factors $(a, c)$ in the effective Lagrangian (3.1), assuming universality in the fermion factors $c_{f} \equiv c$, and assuming that no non-Standard-Model particles contribute to the anomaly factors $c_{g, \gamma}$, which therefore are determined by a combination of the factors $c_{t}=c$ and $a_{W}=a$. In each panel of figure 2 and similar subsequent figures, the more likely regions of parameter space have lighter shading, and the 68, 95 and 99\% CL contours are indicated by dotted, dashed and solid lines, respectively.

We see again in the top row of panels of figure 2 that the data on $H \rightarrow \bar{b} b$ decays (left) and $\tau^{+} \tau^{-}$decays (right) are entirely consistent with the Standard Model predictions $(a, c)=(1,1)$. The region of the $(a, c)$ plane favoured by the $\bar{b} b$ data manifests a correlation between $a$ and $c$ that arises because the dominant production mechanism is associated $V+X$ production, which is $\propto a^{2}$. On the other hand, the region of the $(a, c)$ plane favoured by the $\tau^{+} \tau^{-}$data exhibits a weaker correlation between $a$ and $c$, reflecting the importance of data on production via gluon fusion in this case. As was to be expected from the compilation in figure 1, the $\gamma \gamma$ data displayed in the middle left panel of figure 2 are now compatible with the Standard Model prediction $(a, c)=(1,1)$, following inclusion of the latest CMS result. The data on $H \rightarrow W W^{*}$ (middle right panel of figure 2) and $Z Z^{*}$ decays (bottom left panel) are also entirely consistent with $(a, c)=(1,1)$.

We draw attention to the importance of the 2-jet analyses, which select a VBF-enriched sample, in disfavouring bands of the plots around $c \sim 0$. This effect is very visible in the $\gamma \gamma$ and $W W^{*}$ results displayed in the middle plots. On the other hand, in the $Z Z^{*}$ case the CMS dijet analysis is less powerful, so there is a weaker suppression of the likelihood around $c \sim 0$.

All the above information is combined in the bottom right panel of figure 2, assuming that there are no virtual non-Standard-Model particles contributing to $H \rightarrow \gamma \gamma$ decay or the $\mathrm{Hgg}$ coupling. We note that the global fit is not symmetric between the two possibilities for the sign of $c$ relative to $a$, a feature visible in the middle left panel of figure 2 , and 

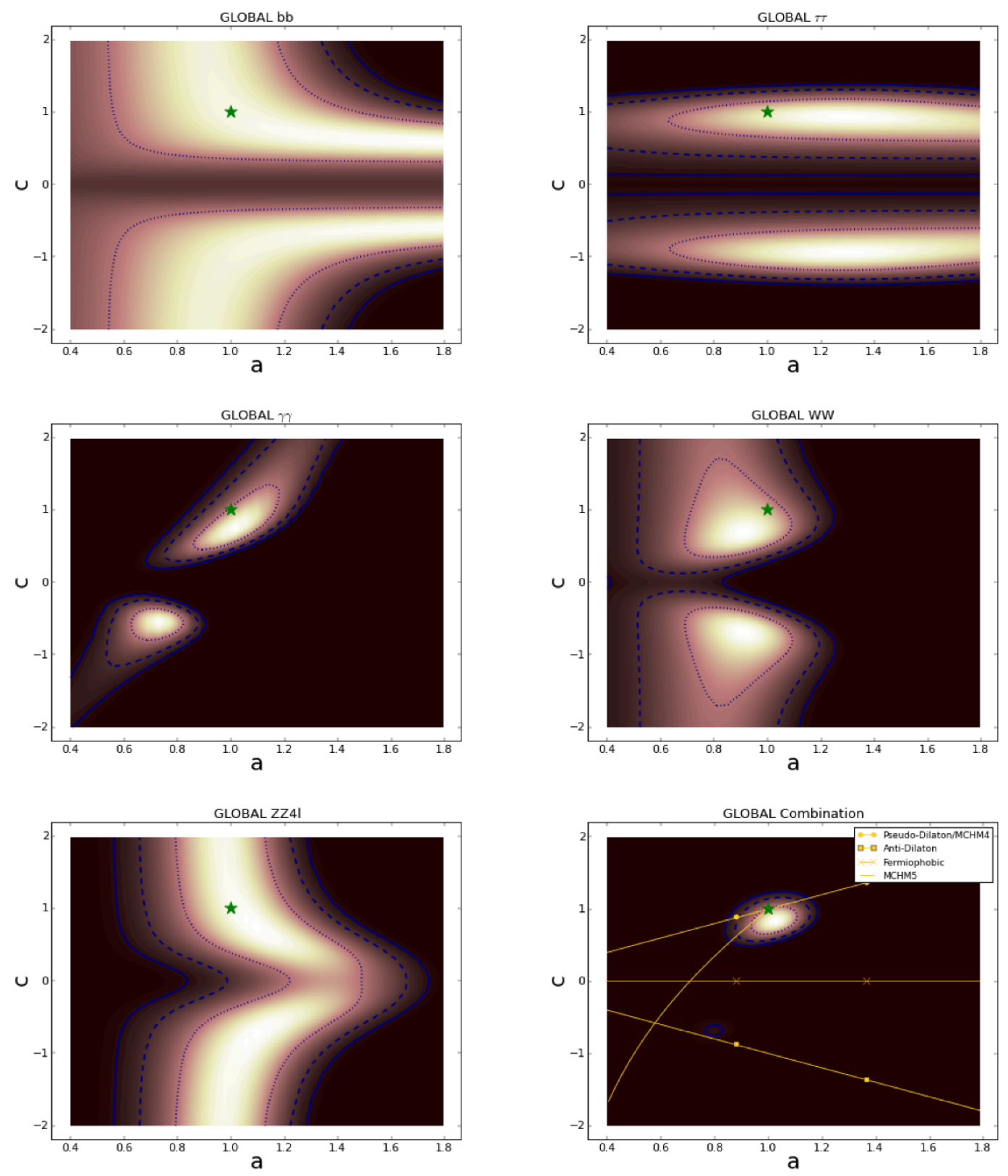

Figure 2. The constraints in the $(a, c)$ plane imposed by the measurements in figure 1 in the $\bar{b} b$ final state (top left), in the $\tau^{+} \tau^{-}$final state (top right), in the $\gamma \gamma$ final state (middle left), in the $W W^{*}$ final state (middle right) and in the $Z Z^{*}$ final state (bottom left). The combination of all these constraints is shown in the bottom right panel.

traceable to the interference between the $t$ quark and $W$ boson loops contributing to the $H \rightarrow \gamma \gamma$ decay amplitude. In the past it has been a common feature of such global fits that they have exhibited two local minima of the likelihood function with opposite signs of $c$ that, because of this asymmetry, were not equivalent but had similar likelihoods [82, 83]. We see in the bottom right panel of figure 2, for the first time a clear preference for the 

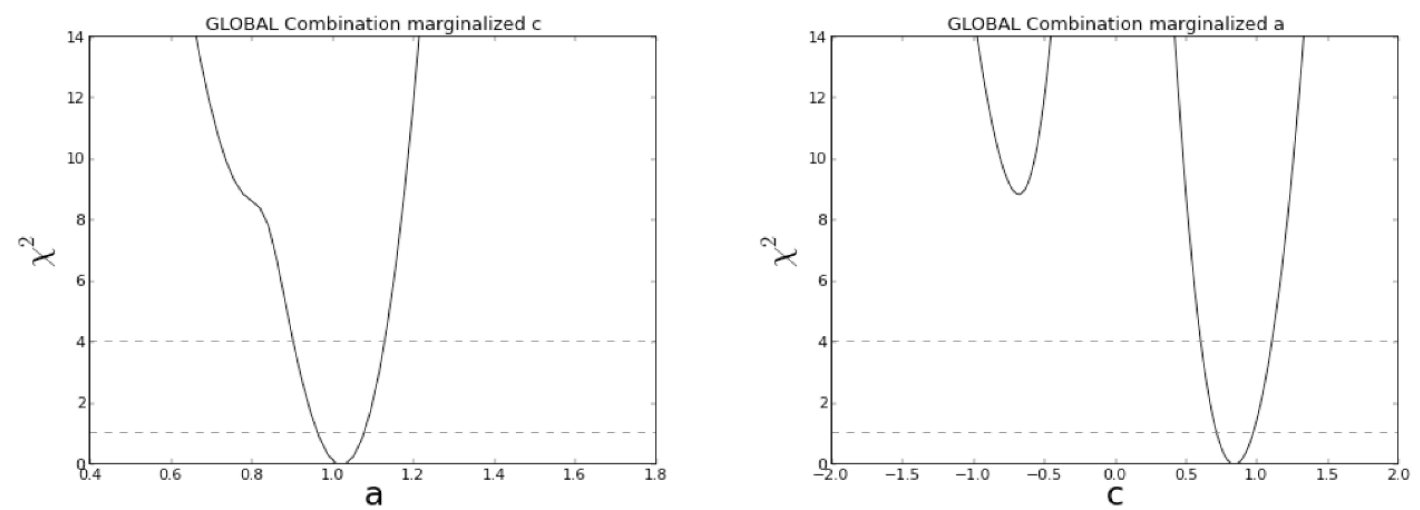

Figure 3. The one-dimensional likelihood functions for the boson coupling parameter $a$ (left panel) and the fermion coupling parameter $c$ (right panel), as obtained by marginalizing over the other parameter in the bottom right panel of figure 2 .

minimum with $c>0$, i.e., the same sign as in the Standard Model.

This feature is also seen clearly in figure 3, where we display in the left panel the one-dimensional likelihood function $\chi^{2}$ for the boson coupling parameter $a$ obtained by marginalizing over the fermion coupling parameter $c$, and in the right panel the onedimensional likelihood function for $c$ obtained by marginalizing over $a$. We see that the fit with $c>0$ is strongly favoured over that with $c<0$, with $\Delta \chi^{2} \sim 9$. The parameters of the global minimum of the $\chi^{2}$ function and their $68 \%$ CL ranges are as follows:

$$
a=1.03 \pm 0.06, c=0.84 \pm 0.15 \text {. }
$$

This preference for $c>0$ is largely driven by the recently-released CMS $\gamma \gamma$ data.

The yellow lines in the bottom right panel of figure 2 correspond to various alternatives to the Standard Model, as discussed above. We see that fermiophobic models (the horizontal line) are very strongly excluded, as are anti-dilaton models in which $c=-a$. On the other hand, dilaton/MCHM4 models with $a=c$ are compatible with the data as long as their common value is close to unity. Likewise, MCHM5 models lying along the curved line are also compatible with the data if their parameters are chosen to give predictions close to the Standard Model.

The fact that, whereas all the direct measurements of $H$ couplings to fermions and massive vector bosons are very compatible with the Standard Model, the coupling to $\gamma \gamma$ was formerly less compatible, has given rise to much speculation that additional virtual particles may be contributing to the factor $c_{\gamma}$ in (3.2). However, the motivation for this speculation has been largely removed by the recent re-evaluation of the $H \rightarrow \gamma \gamma$ decay rate by the CMS Collaboration, which is quite compatible with the Standard Model prediction. The left panel of figure 4 shows the results of a global fit to the anomaly factors $\left(c_{\gamma}, c_{g}\right)$, assuming the Standard Model values $(a, c)=(1,1)$ for the tree-level couplings to massive bosons and fermions. Under this hypothesis, any deviation from $\left(c_{\gamma}, c_{g}\right)=(1,1)$ would be due to new particles beyond the Standard Model. We see explicitly in figure 4 that, while 

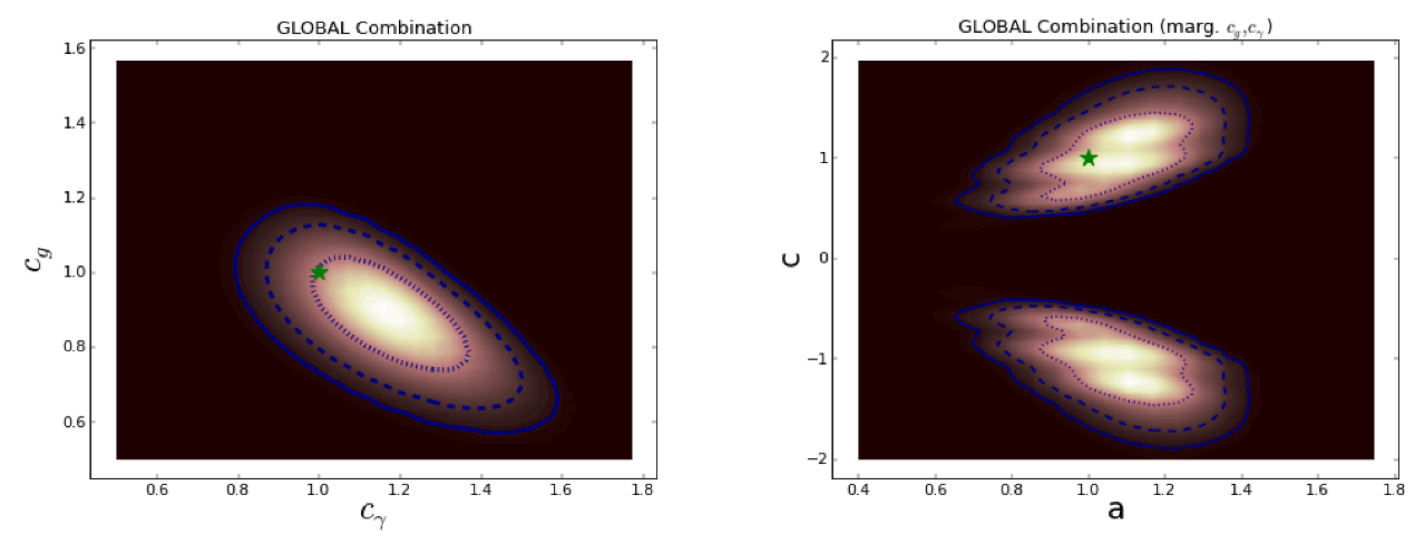

Figure 4. Left: The constraints in the $\left(c_{\gamma}, c_{g}\right)$ plane imposed by the measurements in figure 1, assuming the Standard Model values for the tree-level couplings to massive bosons and fermions, i.e., $a=c=1$. Right: The constraints in the $(a, c)$ plane when marginalizing over $c_{\gamma}$ and $c_{g}$.

there may still be a hint that $c_{\gamma}>1$, the value of $c_{g}$ is completely compatible with the Standard Model. Thus, any set of new particles contributing to $c_{\gamma}$ should be constructed so as not to contribute significantly to $c_{g}$.

The right panel of figure 4 is complementary, showing the constraints in the $(a, c)$ plane after marginalizing over $\left(c_{\gamma}, c_{g}\right)$. Thus it represents the constraints on $a$ and $c$ if no assumption is made about the absence of new particle contributions to the loop amplitudes. In this case, the symmetry between the solutions with $c>0$ and $<0$ is restored, as the $H \rightarrow \gamma \gamma$ decay rate no longer discriminates between them. In this case, the Standard Model values $a=c=1$ are well inside the most favoured region of the $(a, c)$ plane.

We display in the left panel of figure 5 the one-dimensional likelihood function $\chi^{2}$ for the factor $c_{\gamma}$ obtained by marginalizing over $c_{g}$, and in the right panel the one-dimensional likelihood function for $c_{g}$ obtained by marginalizing over $c_{\gamma}$. The central values and the $68 \%$ CL ranges of $c_{\gamma}$ and $c_{g}$ are as follows:

$$
c_{\gamma}=1.18 \pm 0.12, c_{g}=0.88 \pm 0.11,
$$

and the likelihood price for $c_{\gamma}=1$ is $\Delta \chi^{2}=2$, whereas the price for $c_{g}=1$ is $\Delta \chi^{2}=1$.

\section{Probing the mass dependence of Higgs couplings}

We now turn to the results of a global fit using the $(M, \epsilon)$ parameterization (1.1) that probes directly the extent to which the current measurements constrain the $H$ couplings to other particles to be approximately linear: $\epsilon \sim 0$, and the extent to which the mass scaling parameter $M \sim v$. In this limit the Standard Model is recovered in the tree-level approximation. The left panel of figure 6 shows the result of combining the measurements shown in figure 1 in the $(M, \epsilon)$ plane. The horizontal and vertical yellow lines correspond to $\epsilon=0$ and $M=v$, respectively, and the data are quite compatible with these values. The central values and the $68 \%$ CL ranges of $M$ and $\epsilon$ are as follows:

$$
M=244_{-10}^{+20} \mathrm{GeV}, \epsilon=-0.022_{-0.021}^{+0.042},
$$



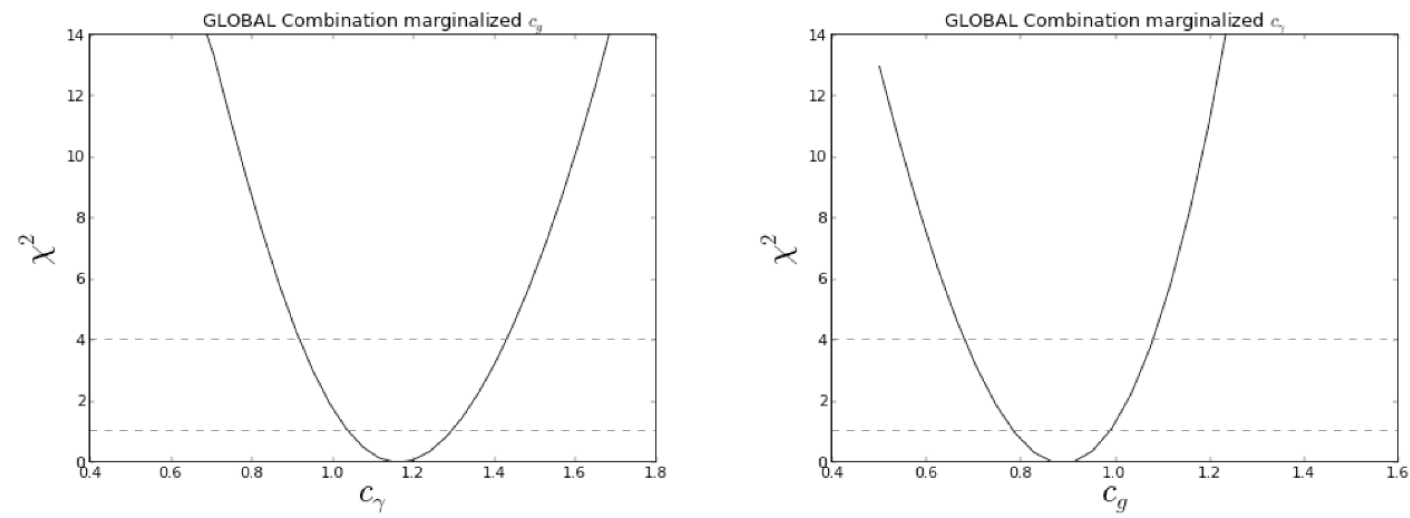

Figure 5. The one-dimensional likelihood functions for $c_{\gamma}$ (left panel) and $c_{g}$ (right panel), as obtained by marginalizing over the other variable in the bottom right panel of figure 4, assuming the Standard Model values for the tree-level couplings to massive bosons and fermions.
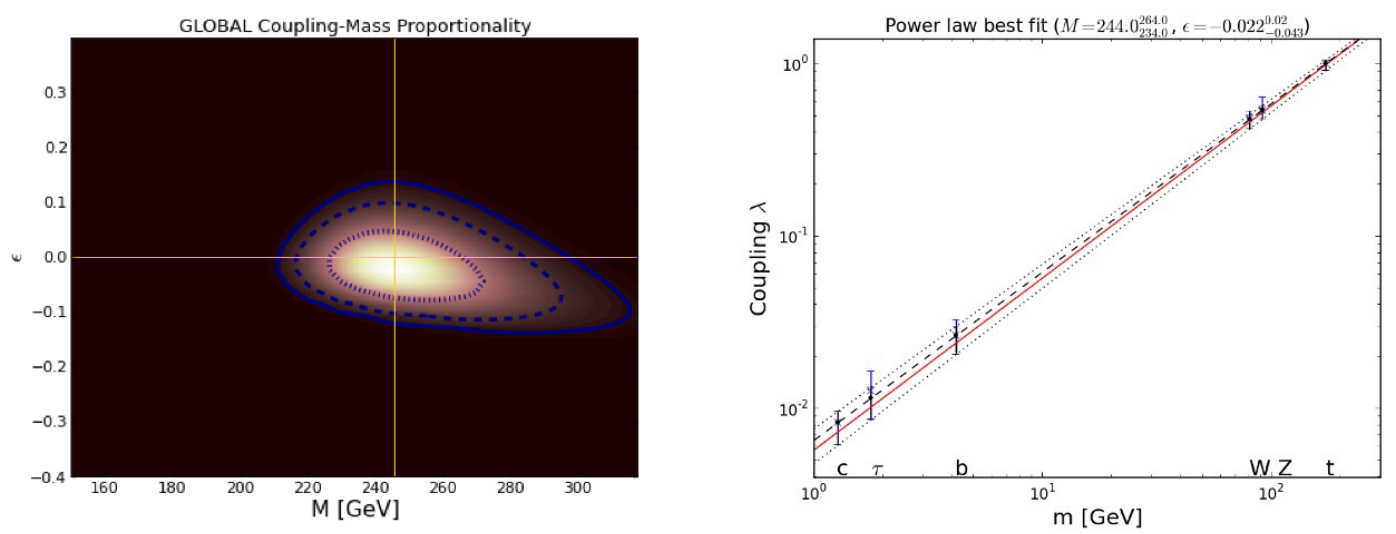

Figure 6. The constraints in the $(M, \epsilon)$ plane imposed by the measurements in figure 1 (left panel) and the strengths of the couplings to different fermion flavours and massive bosons predicted by this two-parameter $(M, \epsilon)$ fit (right panel). In the latter, the red line is the Standard Model prediction, the black dashed line is the best fit, and the dotted lines are the $68 \%$ CL ranges. For each particle species, the black error bar shows the range predicted by the global fit, and the blue error bar shows the range predicted for that coupling if its measurement is omitted from the global fit.

and the likelihood price for $M=246 \mathrm{GeV}$ and $\epsilon=0$ is $\Delta \chi^{2}=0.12$. It is remarkable that the data already constrain the mass dependence of the $H$ couplings to other particles to be linear in their masses to within a few $\%$, and that the mass scaling parameter $M$ is within $10 \%$ of the Standard Model value $v=246 \mathrm{GeV}$. We display in the left panel of figure 7 the one-dimensional likelihood function $\chi^{2}$ for the factor $\epsilon$ obtained by marginalizing over $M$, and in the right panel the one-dimensional likelihood function for $M$ obtained by marginalizing over $\epsilon$.

The right panel of figure 6 displays the mass dependence of the $H$ couplings in a different way, exhibiting explicitly the constraints on the couplings of $H$ to other particles within the parameterization (1.1). The solid red line is the prediction of the Standard 

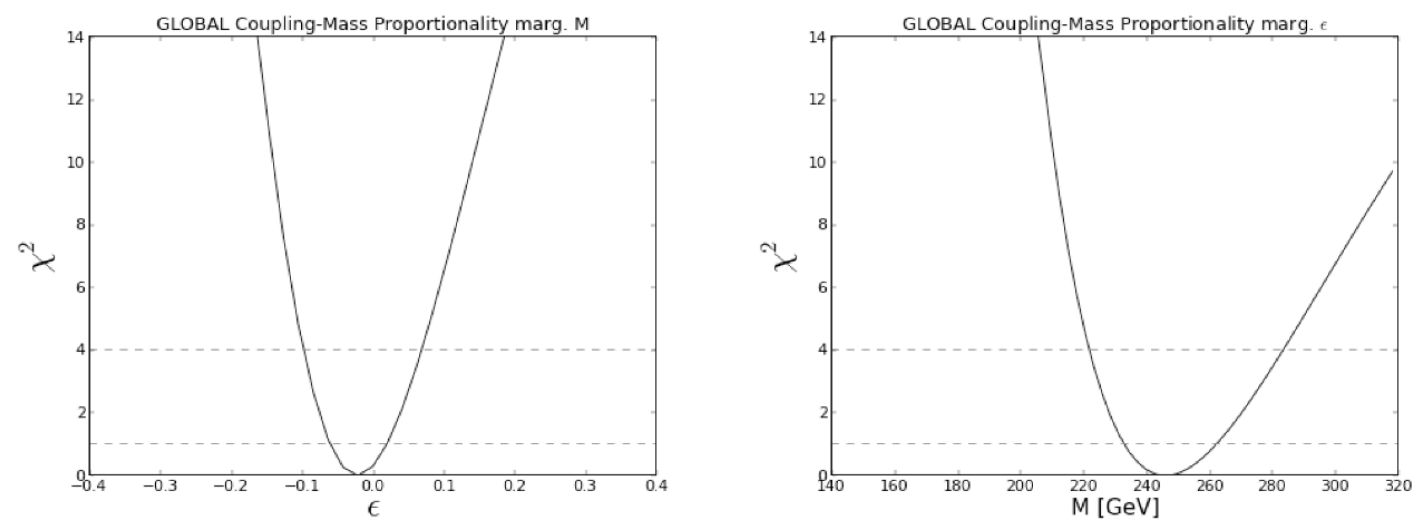

Figure 7. The one-dimensional likelihood functions for $\epsilon$ (left panel) and $M$ (right panel), as obtained by marginalizing over the other variable in the left panel of figure 6 .

Model, $\epsilon=0$ and $M=v$, the black dashed line corresponds to the best-fit values in (4.1), and the dotted lines correspond to their $68 \%$ CL ranges. The black points and vertical error bars are the predictions of the $(M, \epsilon)$ fit for the couplings of $H$ to each of the other particle species: the points lie on the best-fit dashed line and the error bars end on the upper and lower dotted lines. Also shown (in blue) for each particle species is the prediction for its coupling to $H$ if the data on that particular species are omitted from the global fit. In other words, the blue points and error bars represent the predictions for the $H$ coupling to that particle, as derived from the couplings to other particles.

\section{The total Higgs decay rate}

We now discuss the total Higgs decay rate in the two classes of global fit discussed above, assuming that the Higgs has no other decays beyond those in the Standard Model [84, 85]. The left panel of figure 8 displays contours of the Higgs decay rate relative to the Standard Model prediction in the $(a, c)$ plane discussed in section 3. The local $\chi^{2}$ minimum with $c>0$ corresponds to a Higgs decay rate very close to the Standard Model value, whereas the disfavoured 'echo' solution with $c<0$ has a somewhat smaller decay rate. The right panel of figure 8 displays contours of the Higgs decay rate in the $(M, \epsilon)$ plane, where we again see that the best fit has a total decay rate very close to the Standard Model value. We display in figure 9 the one-dimensional likelihood function for the total Higgs decay width relative to its Standard Model value assuming no contributions from non-Standard-Model particles. The solid line is obtained assuming that $a=c$ (or, equivalently, that $\epsilon=0$ but $M$ is free), the dashed line is obtained marginalizing over $(a, c)$, and the dot-dashed line is obtained by marginalizing over $(M, \epsilon)$.

One may also use the current Higgs measurements to constrain the branching ratio for Higgs decays into invisible particles, $B R_{i n v}[86,87]$. This invisible branching ratio factors out of the total decay width as

$$
\Gamma_{\mathrm{Tot}}=\Gamma_{\mathrm{Vis}}+\Gamma_{\mathrm{Inv}}=\left(\frac{R_{\mathrm{Vis}}}{1-B R_{\mathrm{Inv}}}\right) \Gamma_{\mathrm{Tot}}^{\mathrm{SM}}
$$



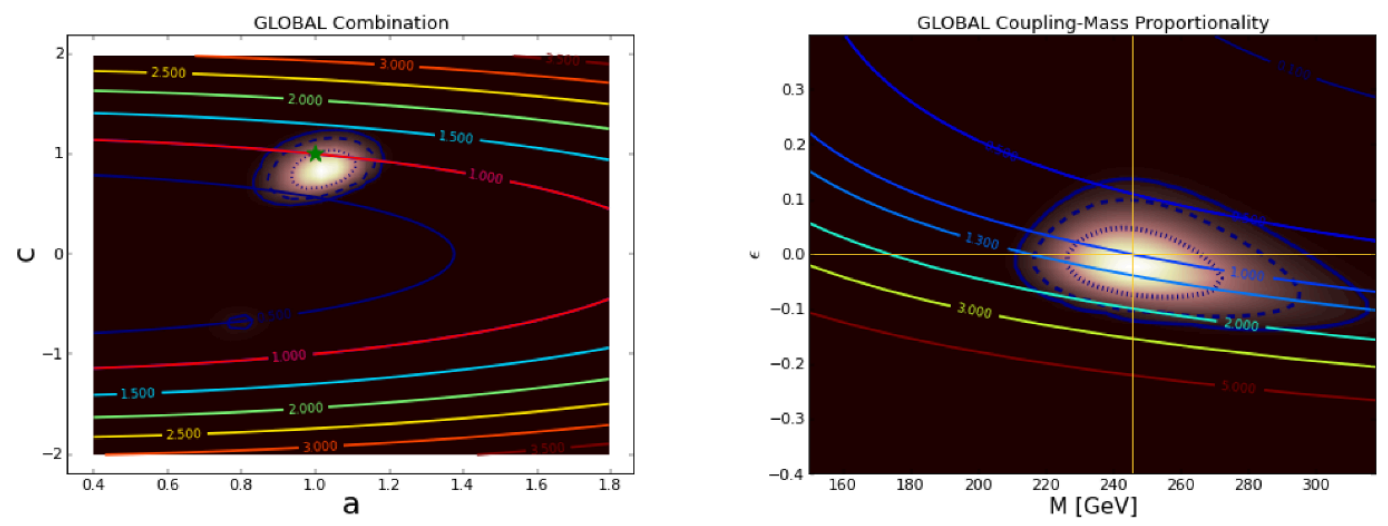

Figure 8. Contours of the total Higgs decay rate relative to the Standard Model prediction in the $(a, c)$ plane shown in the bottom right panel of Fig 2 (left) and the $(M, \epsilon)$ plane shown in the left panel of figure 6 (right).

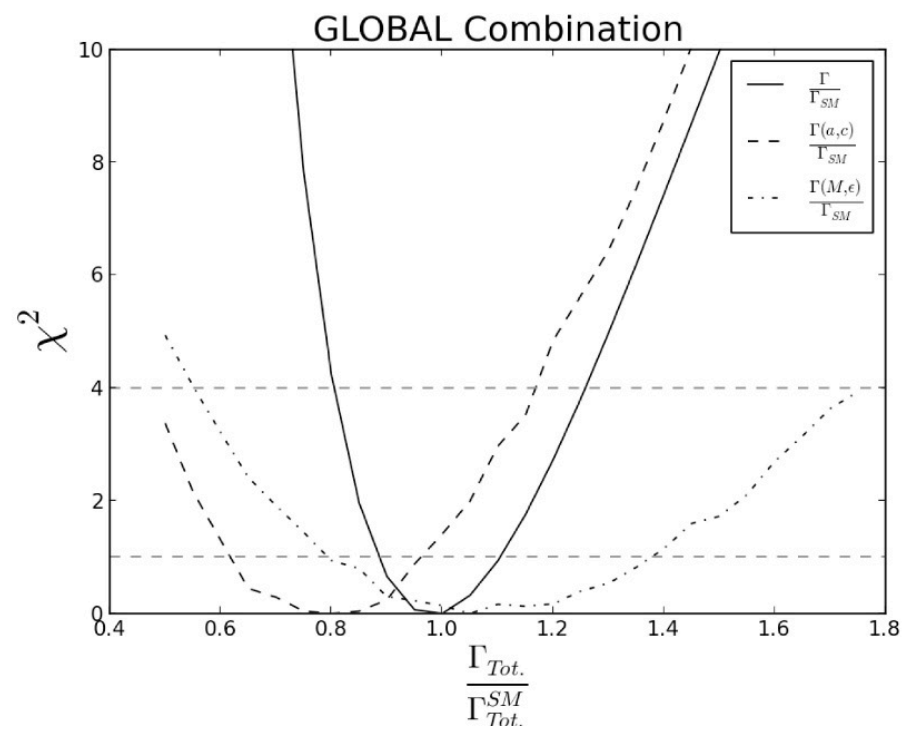

Figure 9. The one-dimensional likelihood function for the total Higgs decay width relative to its value in the Standard Model, $R \equiv \Gamma / \Gamma_{S M}$, assuming decays into Standard Model particles alone and assuming $a=c$ or equivalently $\epsilon=0$ (solid line), marginalizing over $(a, c)$ (dashed line) and marginalizing over $(M, \epsilon)$ (dot-dashed line).

where $R_{\mathrm{Vis}}=\Gamma_{\mathrm{Vis}} / \Gamma_{\mathrm{Tot}}^{\mathrm{SM}}$ is the rescaling factor of the total decay width in the absence of an invisible contribution. Thus we see that an invisible branching ratio acts as a general suppression of all other branching ratios, which could be compensated by non-standard visible Higgs decays.

The left panel of figure 10 displays the $\chi^{2}$ function for $B R_{i n v}$ under various assumptions. The solid line was obtained assuming the Standard Model couplings for visible particles, i.e., $(a, c)=(1,1)$ or equivalently $(M, \epsilon)=(v, 0)$. We see that the best fit has 

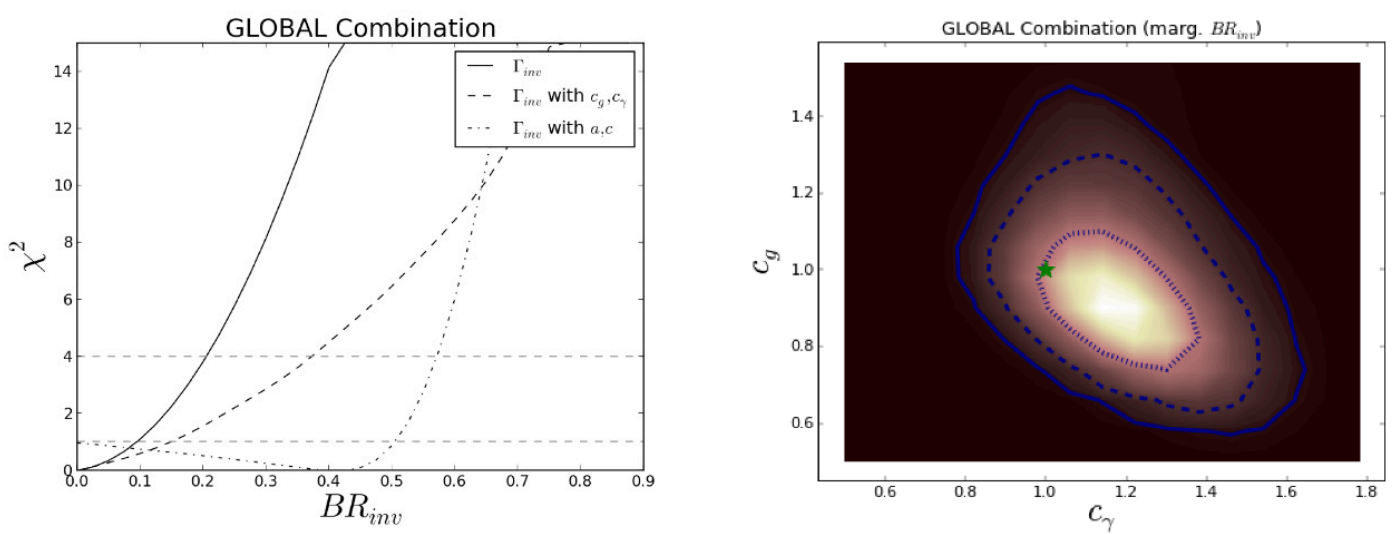

Figure 10. Left: The branching ratio for Higgs decay into invisible particles obtained assuming the Standard Model decay rates for all the visible Higgs decays (solid), marginalizing over $\left(c_{\gamma}, c_{g}\right)$ (dashed) and (a,c) (dot-dashed). Right: The constraints in the $\left(c_{\gamma}, c_{g}\right)$ plane when marginalizing over the invisible branching ration $B R_{i n v}$.

$B R_{i n v}=0$, and that the 68 and $95 \%$ CL limits are 0.09 and 0.21 , respectively. The dot-dashed line was obtained by marginalizing over $(a, c)$, where the shallow minimum at $B R_{\text {inv }} \sim 0.4$ would require $a>1 .{ }^{4}$ Finally, the dashed line was obtained fixing $(a, c)=(1,1)$ (or equivalently $(M, \epsilon)=(v, 0)$ ), but marginalizing over the loop factors $\left(c_{\gamma}, c_{g}\right)$. Conversely, the right panel of figure 10 displays the constraint in the $\left(c_{\gamma}, c_{g}\right)$ plane obtained by marginalizing over $B R_{i n v}$.

\section{Conclusions}

The recent installments of data from the LHC experiments announced in March 2013 impose strong new constraints on the properties and couplings of the $H$ particle, which is a Higgs boson to a high confidence level. The data now constrain this particle to have couplings that differ by only some \% from those of the Higgs boson of the Standard Model. In particular, the relative sign of its couplings to bosons and fermions is fixed for the first time, its couplings to other particles are very close to being linear in their masses, and strong upper limits on invisible Higgs decays can be derived.

The data now impose severe constraints on composite alternatives to the elementary Higgs boson of the Standard Model. However, they do not yet challenge the predictions of supersymmetric models, which typically make predictions much closer to the Standard Model values. We therefore infer that the Higgs coupling measurements, as well as its mass, provide circumstantial support to supersymmetry as opposed to these minimal composite alternatives, though this inference is not conclusive.

It is likely that the first LHC run at 7 and $8 \mathrm{TeV}$ has now yielded most of its Higgs secrets, and we look forward to the next LHC run at higher energy, and its later runs at

\footnotetext{
${ }^{4}$ Constraining $a \leq 1$, as expected in most BSM models, can also lead to interesting upper limits on the invisible Higgs decays [85-87].
} 
significantly higher luminosity. These will provide significant new information about the $H$ particle and constrain further its couplings, as well as providing opportunities to probe directly for other new physics. The LHC will be a hard act to follow.

\section{Acknowledgments}

The work of JE was supported partly by the London Centre for Terauniverse Studies (LCTS), using funding from the European Research Council via the Advanced Investigator Grant 267352. The work of TY was supported by a Graduate Teaching Assistantship from King's College London. JE thanks CERN for kind hospitality.

Open Access. This article is distributed under the terms of the Creative Commons Attribution License which permits any use, distribution and reproduction in any medium, provided the original author(s) and source are credited.

\section{References}

[1] ATLAS collaboration, Observation of a new particle in the search for the Standard Model Higgs boson with the ATLAS detector at the LHC, Phys. Lett. B 716 (2012) 1 [arXiv: 1207.7214] [INSPIRE].

[2] https://twiki.cern.ch/twiki/bin/view/AtlasPublic/HiggsPublicResults.

[3] CMS collaboration, Observation of a new boson at a mass of $125 \mathrm{GeV}$ with the CMS experiment at the LHC, Phys. Lett. B 716 (2012) 30 [arXiv:1207.7235] [INSPIRE].

[4] http://cms.web.cern.ch/org/cms-papers-and-results.

[5] P.W. Higgs, Broken Symmetries and the Masses of Gauge Bosons, Phys. Rev. Lett. 13 (1964) 508 [INSPIRE].

[6] P.W. Higgs, Spontaneous Symmetry Breakdown without Massless Bosons, Phys. Rev. 145 (1966) 1156 [INSPIRE].

[7] J. Ellis, V. Sanz and T. You, Prima Facie Evidence against Spin-Two Higgs Impostors, arXiv: 1211.3068 [INSPIRE].

[8] J. Ellis, V. Sanz and T. You, Associated Production Evidence against Higgs Impostors and Anomalous Couplings, arXiv:1303.0208 [INSPIRE].

[9] ATLAS collaboration, Study of the spin of the Higgs-like boson in the two photon decay channel using $20.7 \mathrm{fb}^{-1}$ of pp collisions collected at $\sqrt{s}=8 \mathrm{TeV}$ with the ATLAS detector, ATLAS-CONF-2013-029 (2013).

[10] M. Chen on behalf of the CMS collaboration, Combination and Standard Model Scalar Boson Properties in CMS, talk given at the Rencontres de Moriond Electroweak Session (2013), https://indico.in2p3.fr/getFile.py/access?contribId=15\&sessionId=6\&resId= o\&material $I d=$ slides\&conf $I d=7411$.

[11] T. Adye on behalf of the ATLAS collaboration, Measurement of Higgs Boson Properties in ATLAS, Talk given at the Rencontres de Moriond Electroweak Session (2013), http://moriond.in2p3.fr/QCD/2013/ThursdayMorning/Adye.pdf. 
[12] CMS collaboration, S. Chatrchyan et al., On the mass and spin-parity of the Higgs boson candidate via its decays to Z boson pairs, Phys. Rev. Lett. 110 (2013) 081803 [arXiv: 1212.6639] [INSPIRE].

[13] M. Baak et al., Updated Status of the Global Electroweak Fit and Constraints on New Physics, Eur. Phys. J. C 72 (2012) 2003 [arXiv:1107.0975] [INSPIRE].

[14] J.R. Ellis, G. Ridolfi and F. Zwirner, Radiative corrections to the masses of supersymmetric Higgs bosons, Phys. Lett. B 257 (1991) 83 [INSPIRE].

[15] Y. Okada, M. Yamaguchi and T. Yanagida, Renormalization group analysis on the Higgs mass in the softly broken supersymmetric standard model, Phys. Lett. B 262 (1991) 54 [INSPIRE].

[16] H.E. Haber and R. Hempfling, Can the mass of the lightest Higgs boson of the minimal supersymmetric model be larger than m(Z)?, Phys. Rev. Lett. 66 (1991) 1815 [INSPIRE].

[17] Hadron Collider Physics Symposium 2012, http://www.icepp.s.u-tokyo.ac.jp/hcp2012/.

[18] M. Kado on behalf of the ATLAS collaboration, Physics Jamboree at CERN, Dec. 13th, 2012, http://indico. cern. ch/getFile.py/access?resId=0\&materialId= slides\&contribId=0\&session $I d=0 \&$ subCont $I d=3 \& \operatorname{con} f I d=218449$.

[19] S. Bolognesi on behalf of the CMS collaboration, Physics Jamboree at CERN, Dec. 13th, 2012, http://indico. cern. ch/getFile.py/access?resId=0\&materialld= slides\&contribId=0\&session $I d=0 \&$ subCont $I d=2 \&$ conf $I d=218449$.

[20] Rencontres de Moriond 2013 Electroweak Session, https://indico.in2p3.fr/conferenceDisplay . py? conf Id=7411.

[21] Aspen 2013 - Higgs Quo Vadis, http: //indico. cern. ch/conferenceDisplay . py? conf Id=202554.

[22] Rencontres de Moriond 2013 QCD Session, http://moriond.in2p3.fr/QCD/2013/MorQCD13Prog.html.

[23] D. Carmi, A. Falkowski, E. Kuflik and T. Volansky, Interpreting LHC Higgs Results from Natural New Physics Perspective, JHEP 07 (2012) 136 [arXiv:1202.3144] [INSPIRE].

[24] A. Azatov, R. Contino and J. Galloway, Model-Independent Bounds on a Light Higgs, JHEP 04 (2012) 127 [Erratum ibid. 1304 (2013) 140] [arXiv: 1202.3415] [INSPIRE].

[25] J. Espinosa, C. Grojean, M. Muhlleitner and M. Trott, Fingerprinting Higgs Suspects at the LHC, JHEP 05 (2012) 097 [arXiv: 1202.3697] [INSPIRE].

[26] P.P. Giardino, K. Kannike, M. Raidal and A. Strumia, Reconstructing Higgs boson properties from the LHC and Tevatron data, JHEP 06 (2012) 117 [arXiv: 1203.4254] [INSPIRE].

[27] T. Li, X. Wan, Y.-k. Wang and S.-h. Zhu, Constraints on the Universal Varying Yukawa Couplings: from SM-like to Fermiophobic, JHEP 09 (2012) 086 [arXiv:1203.5083] [INSPIRE].

[28] M. Rauch, Determination of Higgs-boson couplings (SFitter), arXiv:1203.6826 [INSPIRE].

[29] J. Ellis and T. You, Global Analysis of Experimental Constraints on a Possible Higgs-Like Particle with Mass 125 GeV, JHEP 06 (2012) 140 [arXiv:1204.0464] [INSPIRE].

[30] A. Azatov et al., Determining Higgs couplings with a model-independent analysis of $h \rightarrow \gamma \gamma$, JHEP 06 (2012) 134 [arXiv:1204.4817] [INSPIRE]. 
[31] M. Klute, R. Lafaye, T. Plehn, M. Rauch and D. Zerwas, Measuring Higgs Couplings from LHC Data, Phys. Rev. Lett. 109 (2012) 101801 [arXiv:1205.2699] [INSPIRE].

[32] L. Wang and X.-F. Han, The recent Higgs boson data and Higgs triplet model with vector-like quark, Phys. Rev. D 86 (2012) 095007 [arXiv:1206.1673] [InSPIRE].

[33] D. Carmi, A. Falkowski, E. Kuflik and T. Volansky, Interpreting the Higgs, arXiv:1206.4201 [INSPIRE].

[34] M.J. Dolan, C. Englert and M. Spannowsky, Higgs self-coupling measurements at the LHC, JHEP 10 (2012) 112 [arXiv:1206.5001] [INSPIRE].

[35] J. Chang, K. Cheung, P.-Y. Tseng and T.-C. Yuan, Distinguishing Various Models of the 125 GeV Boson in Vector Boson Fusion, JHEP 12 (2012) 058 [arXiv: 1206.5853] [INSPIRE].

[36] S. Chang, C.A. Newby, N. Raj and C. Wanotayaroj, Revisiting Theories with Enhanced Higgs Couplings to Weak Gauge Bosons, Phys. Rev. D 86 (2012) 095015 [arXiv:1207.0493] [INSPIRE].

[37] I. Low, J. Lykken and G. Shaughnessy, Have We Observed the Higgs (Imposter)?, Phys. Rev. D 86 (2012) 093012 [arXiv:1207.1093] [INSPIRE].

[38] T. Corbett, O. Eboli, J. Gonzalez-Fraile and M. Gonzalez-Garcia, Constraining anomalous Higgs interactions, Phys. Rev. D 86 (2012) 075013 [arXiv: 1207.1344] [InSPIRE].

[39] P.P. Giardino, K. Kannike, M. Raidal and A. Strumia, Is the resonance at $125 \mathrm{GeV}$ the Higgs boson?, Phys. Lett. B 718 (2012) 469 [arXiv:1207.1347] [INSPIRE].

[40] M. Montull and F. Riva, Higgs discovery: the beginning or the end of natural EWSB?, JHEP 11 (2012) 018 [arXiv: 1207.1716] [INSPIRE].

[41] J. Espinosa, C. Grojean, M. Muhlleitner and M. Trott, First Glimpses at Higgs' face, JHEP 12 (2012) 045 [arXiv: 1207.1717] [INSPIRE].

[42] D. Carmi, A. Falkowski, E. Kuflik, T. Volansky and J. Zupan, Higgs After the Discovery: A Status Report, JHEP 10 (2012) 196 [arXiv:1207.1718] [InSPIRE].

[43] S. Banerjee, S. Mukhopadhyay and B. Mukhopadhyaya, New Higgs interactions and recent data from the LHC and the Tevatron, JHEP 10 (2012) 062 [arXiv:1207.3588] [INSPIRE].

[44] F. Bonnet, T. Ota, M. Rauch and W. Winter, Interpretation of precision tests in the Higgs sector in terms of physics beyond the Standard Model, Phys. Rev. D 86 (2012) 093014 [arXiv: 1207.4599] [INSPIRE].

[45] T. Plehn and M. Rauch, Higgs Couplings after the Discovery, Europhys. Lett. 100 (2012) 11002 [arXiv: 1207.6108] [INSPIRE].

[46] A. Djouadi, Precision Higgs coupling measurements at the LHC through ratios of production cross sections, arXiv:1208.3436 [INSPIRE].

[47] B. Batell, S. Gori and L.T. Wang, Higgs couplings and precision electroweak data, arXiv:1209.6832.

[48] G. Moreau, Constraining extra-fermion(s) from the Higgs boson data, Phys. Rev. D 87 (2013) 015027 [arXiv: 1210.3977] [INSPIRE].

[49] G. Cacciapaglia, A. Deandrea, G.D. La Rochelle and J.-B. Flament, Higgs couplings beyond the Standard Model, JHEP 03 (2013) 029 [arXiv: 1210.8120] [INSPIRE].

[50] E. Masso and V. Sanz, Limits on Anomalous Couplings of the Higgs to Electroweak Gauge Bosons from LEP and LHC, Phys. Rev. D 87 (2013) 033001 [arXiv:1211.1320] [InSPIRE]. 
[51] T. Corbett, O. Eboli, J. Gonzalez-Fraile and M. Gonzalez-Garcia, Robust Determination of the Higgs Couplings: Power to the Data, Phys. Rev. D 87 (2013) 015022 [arXiv:1211.4580] [INSPIRE].

[52] R.T. D'Agnolo, E. Kuflik and M. Zanetti, Fitting the Higgs to Natural SUSY, JHEP 03 (2013) 043 [arXiv: 1212.1165] [InSPIRE].

[53] A. Azatov and J. Galloway, Electroweak Symmetry Breaking and the Higgs Boson: Confronting Theories at Colliders, Int. J. Mod. Phys. A 28 (2013) 1330004 [arXiv: 1212.1380] [INSPIRE].

[54] G. Bhattacharyya, D. Das and P.B. Pal, Modified Higgs couplings and unitarity violation, Phys. Rev. D 87 (2013) 011702 [arXiv: 1212.4651] [INSPIRE].

[55] D. Choudhury, R. Islam, A. Kundu and B. Mukhopadhyaya, Anomalous Higgs Couplings as a Window to New Physics, arXiv: 1212.4652 [INSPIRE].

[56] R.S. Gupta, M. Montull and F. Riva, SUSY Faces its Higgs Couplings, JHEP 04 (2013) 132 [arXiv: 1212.5240] [INSPIRE].

[57] G. Bélanger, B. Dumont, U. Ellwanger, J. Gunion and S. Kraml, Higgs Couplings at the End of 2012, JHEP 02 (2013) 053 [arXiv: 1212.5244] [INSPIRE].

[58] K. Cheung, J.S. Lee and P.-Y. Tseng, Higgs Precision (Higgcision) Era begins, JHEP 05 (2013) 134 [arXiv: 1302.3794] [INSPIRE].

[59] J. Ellis and T. You, Global Analysis of the Higgs Candidate with Mass 125 GeV, JHEP 09 (2012) 123 [arXiv:1207.1693] [INSPIRE].

[60] A. Falkowski, F. Riva and A. Urbano, Higgs at Last, arXiv:1303:1812.

[61] P.P. Giardino, K. Kannike, I. Masina, M. Raidal and A. Strumia, The universal Higgs fit, arXiv:1303.3570 [INSPIRE].

[62] ATLAS collaboration, Combined coupling measurements of the Higgs-like boson with the ATLAS detector using up to $25 \mathrm{fb}^{-1}$ of proton-proton collision data, ATLAS-CONF-2013-034 (2013).

[63] CMS collaboration, CMS Physics Analysis Summary, HIG-13-005-pas http://cds . cern.ch/record/1542387/files/HIG-13-005-pas.pdf.

[64] LHC. Higgs Cross section Working Group, LHC HXSWG interim recommendations to explore the coupling structure of a Higgs-like particle, arXiv:1209.0040 [INSPIRE].

[65] F. Englert and R. Brout, Broken Symmetry and the Mass of Gauge Vector Mesons, Phys. Rev. Lett. 13 (1964) 321 [INSPIRE].

[66] P.W. Higgs, Broken symmetries, massless particles and gauge fields, Phys. Lett. 12 (1964) 132 [INSPIRE].

[67] G. Guralnik, C. Hagen and T. Kibble, Global Conservation Laws and Massless Particles, Phys. Rev. Lett. 13 (1964) 585 [InSPIRE].

[68] W. Yao on behalf of the TeVatron collaboration, Tevatron Combination and BEH properties, Talk given at the Rencontres de Moriond Electroweak Session (2013), https://indico.in2p3.fr/getFile.py/access? contribId=69\&sessionId=6\&res Id= O\&materialld=slides\&conf $\mathrm{Id}=7411$.

[69] CMS collaboration, Update on the search for the standard model Higgs boson in pp collisions at the $L H C$ decaying to $W+W$ ? in the fully leptonic final state, http://cds. cern. ch/record/1449158/files/HIG-12-003-pas.pdf. 
[70] CMS collaboration, Evidence for a particle decaying to $W+W$ ? in the fully leptonic final state in a standard model Higgs boson search in pp collisions at the $\mathrm{LHC}$, http://cds . cern. ch/record/1493602/files/HIG-12-042-pas . pdf.

[71] CMS collaboration, Observation of a new boson with a mass near 125 GeV, http://cds. cern.ch/record/1460438/files/HIG-12-020-pas.pdf.

[72] ATLAS collaboration, Measurements of the properties of the Higgs-like boson in the $W W^{(*)} \rightarrow \ell \nu \ell \nu$ decay channel with the ATLAS detector using $25 \mathrm{fb}^{-1}$ of proton-proton collision data, ATLAS-CONF-2013-030 (2013).

[73] CMS collaboration, Combination of standard model Higgs boson searches and measurements of the properties of the new boson with a mass near $125 \mathrm{GeV}$, http://cds.cern.ch/record/1494149/files/HIG-12-045-pas.pdf.

[74] ATLAS collaboration, Search for the Standard Model Higgs boson in produced in association with a vector boson and decaying to bottom quarks with the ATLAS detector, ATLAS-CONF-2012-161 (2012).

[75] ATLAS collaboration, Search for the Standard Model Higgs boson in $H \rightarrow \tau^{+} \tau^{-}$decays in proton-proton collisions with the ATLAS detector, ATLAS-CONF-2012-160 (2012).

[76] CMS collaboration, Search for the standard model Higgs boson decaying to tau pairs, http://cds.cern.ch/record/1493615/files/HIG-12-043-pas .pdf.

[77] CMS collaboration, Evidence for a new state decaying into two photons in the search for the standard model Higgs boson in pp collisions, http://cds. cern.ch/record/1460419/files/HIG-12-015-pas.pdf.

[78] G. Giudice, C. Grojean, A. Pomarol and R. Rattazzi, The Strongly-Interacting Light Higgs, JHEP 06 (2007) 045 [hep-ph/0703164] [INSPIRE].

[79] R. Contino, C. Grojean, M. Moretti, F. Piccinini and R. Rattazzi, Strong Double Higgs Production at the LHC, JHEP 05 (2010) 089 [arXiv: 1002.1011] [INSPIRE].

[80] R. Contino, The Higgs as a Composite Nambu-Goldstone Boson, arXiv:1005.4269 [INSPIRE].

[81] R. Grober and M. Muhlleitner, Composite Higgs Boson Pair Production at the LHC, JHEP 06 (2011) 020 [arXiv: 1012.1562] [INSPIRE].

[82] S. Biswas, E. Gabrielli and B. Mele, Single top and Higgs associated production as a probe of the Htt coupling sign at the LHC, JHEP 01 (2013) 088 [arXiv:1211.0499] [INSPIRE].

[83] M. Farina, C. Grojean, F. Maltoni, E. Salvioni and A. Thamm, Lifting degeneracies in Higgs couplings using single top production in association with a Higgs boson, JHEP 05 (2013) 022 [arXiv: 1211.3736] [INSPIRE].

[84] V. Barger, M. Ishida and W.-Y. Keung, Total Width of 125 GeV Higgs Boson, Phys. Rev. Lett. 108 (2012) 261801 [arXiv:1203.3456] [INSPIRE].

[85] B.A. Dobrescu and J.D. Lykken, Coupling spans of the Higgs-like boson, JHEP 02 (2013) 073 [arXiv: 1210.3342] [INSPIRE].

[86] J.R. Espinosa, M. Muhlleitner, C. Grojean and M. Trott, Probing for Invisible Higgs Decays with Global Fits, JHEP 09 (2012) 126 [arXiv:1205.6790] [INSPIRE].

[87] G. Bélanger, B. Dumont, U. Ellwanger, J. Gunion and S. Kraml, Status of invisible Higgs decays, Phys. Lett. B 723 (2013) 340 [arXiv:1302.5694] [InSPIRE]. 